\title{
INTERACTION HOULE-COURANT EN BATHYMETRIE VARIABLE VIA UNE APPROCHE DE TYPE BOUSSINESQ
}

\section{WAVE-CURRENT INTERACTION ON VARYING BATHYMETRY WITH A BOUSSINESQ APPROACH}

\author{
F. GUINOT*, M. LE BOULLUEC ${ }^{*}$, V. REY ${ }^{*}$ \\ * Hydrodynamique et Océanométéo, Ifremer, Centre de Brest, BP 70, 29280 Plouzané \\ ${ }^{* *}$ LSEET-LEPI, Université du Sud Toulon-Var, BP 2013283957 La Garde Cedex
}

\section{Résumé}

La modélisation de la cinématique de sites à forts courants exposés à la houle requiert une approche équilibrée entre complexité du modèle et finesse de la description recherchée.

Afin de représenter la cinématique de l'écoulement à l'échelle de chaque vague, une formulation de type Boussinesq originale permettant la présence de bathymétrie variable est employée. Celle-ci doit être adaptée afin de prendre en compte les effets du courant. Les résultats sur le décalage Doppler, le blocage des vagues ainsi que sur les profils de vitesses sont présentés dans un cas bidimensionnel.

\section{Summary}

Modeling wave-current interaction needs a method combining accurate kinematics and reasonable computing efforts. Refined waves kinematics are described by a Boussinesq-type approach allowing a variable bathymetry. This method is adapted to take current effects into account. Results on Doppler shift, waves blocking and velocity profile are reported in a twodimensional case.

Mots clés : Interaction houle-courant, approche de Boussinesq, profil de vitesse Key words : Wave-current interaction, Boussinesq approach, velocity profile 


\section{L-INTRODUCTION}

De nouveaux systèmes de conversion de l'énergie cinétique des courants marins ont récemment été développés. Ces dispositifs, conçus pour fonctionner dans des courants incidents idéalement uniformes et stationnaires, sont appelés à être soumis à des écoulements complexes et fluctuants résultant de la combinaison de la houle et de forts courants interagissant sur une bathymétrie inégale. Il est donc nécessaire de bien connaître ces phénomènes d'interaction pour à la fois appréhender la production réelle d'un dispositif et permettre son dimensionnement structurel. Un outil de modélisation est développé en ce sens. Les interactions houle courant peuvent être étudiées de diverses manières selon l'échelle requise.

A grande échelle, des modèles de circulation générale couplés à des modèles spectraux de houle permettent d'étudier l'évolution d'un champ de vagues en présence d'un courant [1].

Peu d'entre eux traitent des modifications du champ de courant par la houle et ils ne fournissent pas directement d'informations sur la cinématique interne mais peuvent fournir des conditions aux limites à des modèles d'échelles plus locales.

A petite échelle, les effets visqueux et turbulents au travers de la résolution des équations de Navier-Stokes peuvent être pris en compte tant pour la modélisation de courants seuls [2] que de l'interaction houle et courant $[3,4]$, cependant les temps de calcul et le pas en espace ne permettent de traiter que des domaines très petits. Une étude spécifique [12] a démontré la possibilité d'appliquer un code industriel comme Fluent à la modélisation d'une hydrolienne en présence de courant. Ainsi un modèle capable de traiter des domaines de taille intermédiaire est indispensable afin de fournir des profils d'écoulement réalistes en entrée de ce type de code.

Un modèle fondé sur une approche de type Boussinesq originale a été choisi afin de faire le lien entre les modèles grandes échelles (de l'ordre du km) et petites échelles (de l'ordre du mètre).

La formulation choisie est basée sur l'approche de Madsen et al. ([6] et [9]) qui permet de traiter la propagation de houle en présence une bathymétrie fortement variable. Les propriétés de dispersion, de shoaling, d'interaction non linéaire hors courant sont très bien reproduites par cette approche mais ne sont pas développées dans cet article.

\section{Ш-EQUATIONS DU MODELE}

On considère l'écoulement irrotationnel d'un fluide à surface libre incompressible et nonvisqueux, référencé par un système de coordonnées cartésiennes dont les axes $\mathbf{x}$ et $\mathbf{y}$ sont situés dans le plan de la surface libre au repos $z=0$ et l'axe $\mathbf{z}$ orienté verticalement vers le haut. Le fluide est limité par un fond variable en $z=-h(x, y)$ et par la surface libre en $z=\eta(x, y, t)$. Le champ des vitesses dérive d'un potentiel $\phi$ de la façon suivante :

$$
u=\nabla \phi, \quad \nabla \equiv\left(\frac{\partial}{\partial x}, \frac{\partial}{\partial y}\right), \quad w=\frac{\partial \phi}{\partial z}
$$

D'après Zakharov [5], les conditions dynamique et cinématique peuvent être exprimées en terme de variables de vitesse définies directement à la surface libre :

$$
\begin{aligned}
& \eta_{\mathrm{t}}+\tilde{u} \cdot \nabla \eta-\widetilde{w}=0 \\
& \widetilde{U}_{\mathrm{t}}+\mathrm{g} \nabla \eta+\frac{1}{2} \nabla\left(\widetilde{U}^{2}-\frac{\widetilde{w}^{2}}{2}\left(1+(\nabla \eta)^{2}\right)=0\right.
\end{aligned}
$$

avec

$$
\begin{aligned}
& \widetilde{u}=(\nabla \phi)_{z=\eta} \\
& \widetilde{w}=\left(\phi_{z}\right)_{z=\eta} \\
& \widetilde{U}=\nabla \widetilde{\phi}=\widetilde{u}+\widetilde{w} \nabla \eta
\end{aligned}
$$

Ces deux équations non-linéaires exactes assurent la marche en temps dans le modèle. 
Le potentiel doit par ailleurs vérifier l'équation de Laplace au sein du fluide et la condition cinématique de glissement sur le fond :

$$
\begin{array}{ll}
\Delta \phi=0 & (4) \\
w_{b}+\nabla h \cdot u_{b}=0 & \text { (5) }
\end{array} \quad \text { avec } w_{b}=w(x, y,-h, t) \text { et } u_{b}=u(x, y,-h, t)
$$

La résolution de l'équation de Laplace s'appuie sur la méthode suivante ([6] et [7]).

A partir d'un développement en série du potentiel des vitesses $\phi(x, y, z, t)=\left.\sum_{n=0}^{\infty} \frac{z^{n}}{n !} \frac{\partial^{n} \phi}{\partial z^{n}}\right|_{z=0}$ une solution exacte de l'équation de Laplace dans le fluide peut être exprimée par :

$$
\begin{array}{lrl}
u(x, y, z, t)=\cos (z \nabla) u_{0}+\sin (z \nabla) w_{0} \text { avec } & \cos (z \nabla)=\sum_{n=0}^{\infty}(-1)^{n} \frac{z^{2 n}}{(2 n !)} \nabla^{2 n} \\
w(x, y, z, t)=\cos (z \nabla) w_{0}-\sin (z \nabla) u_{0} & \sin (z \nabla)=\sum_{n=0}^{\infty}(-1)^{n} \frac{z^{2 n+1}}{(2 n+1 !)} \nabla^{2 n+1}
\end{array}
$$

$w_{0}$ et $u_{0}$ étant respectivement les vitesses verticales et horizontales définies au niveau de la surface libre au repos. Cependant le fait de conserver des variables de vitesse définies au niveau d'eau moyen entraîne une mauvaise reproduction des profils verticaux des vitesses dans la colonne d'eau. On exprime alors les équations précédentes à un niveau d'eau arbitraire supposé être une fraction constante de la hauteur d'eau au repos :

$\hat{z}=-\sigma h(x, y)$

Après inversion on peux exprimer $u_{0}$ et $w_{0}$ en fonction de $\hat{\mathrm{u}}$ et $\hat{\mathrm{w}}$ et finalement on obtient : $u(x, y, z, t)=\cos ((z-\hat{z}) \nabla) \hat{\mathrm{u}}+\sin ((z-\hat{z}) \nabla \hat{\mathrm{w}})+\nabla \hat{z} \ldots$.

$w(x, y, z, t)=\cos ((z-\hat{z}) \nabla) \hat{\mathrm{w}}-\sin ((z-\hat{z}) \nabla \hat{\mathrm{u}})+\nabla \hat{z} \ldots$.

On constate qu'en choisissant $\sigma \approx 0.5$, on minimise les erreurs commises sur le profil de vitesses. Cependant l'introduction de nouvelles variables va permettre d'améliorer grandement la précision des équations en introduisant des approximations de Padé au fond et au niveau d'eau moyen $\mathrm{z}=0$. Ces nouvelles variables sont définies par :

$\hat{u} \equiv L(\hat{z} \nabla) \hat{u}^{*} \quad$ avec $L$ un opérateur linéaire de la forme $L(\hat{z} \nabla) \equiv \sum_{\mathrm{n}=0}^{2 \mathrm{~N}} \lambda_{2 n} \hat{z}^{2 n} \nabla^{2 n}$

$\hat{w} \equiv L(\hat{z} \nabla) \hat{w}^{*}$

Les coefficients $\lambda_{2 n}$ sont déterminés de manière à obtenir des approximations de Padé $[4,4]$ au niveau d'eau moyen, doublant ainsi la précision par rapport à l'ordre des dérivées incluses. La formulation finale des vitesses s'écrit alors sous cette forme :

$$
\begin{aligned}
& u(x, y, z, t)=J_{I} \hat{u}^{*}+J_{I I} \hat{w}^{*} \\
& w(x, y, z, t)=J_{I} \hat{w}^{*}-J_{I I} \hat{u}^{*}
\end{aligned}
$$

avec $J_{I}$ et $J_{I I}$ des opérateurs linéaires fonctions de $z, \hat{z}$ et $\nabla$ (Furhman [8] ou Madsen et al. [9]).

Enfin en insérant ces équations dans l'équation cinématique au fond on obtient :

$$
J_{I}(\mathrm{z}=-\mathrm{h}) \hat{w}^{*}-J_{I I}(\mathrm{z}=-\mathrm{h}) \hat{u}^{*}+\nabla h\left[J_{I}(\mathrm{z}=-\mathrm{h}) \hat{u}^{*}+J_{I I}(\mathrm{z}=-\mathrm{h}) \hat{w}^{*}\right]=0
$$

\section{Ш - LE MODELE NUMERIQUE}

\section{III.1 Caractéristiques générales}

Un modèle numérique en 2 dimensions (une dimension horizontale) fondé sur les équations précédentes a été développé. L'intégration en temps se fait via un schéma de type RungeKutta d'ordre 4 . A chaque instant il faut déterminer $\widetilde{w}$ afin de pouvoir résoudre les équations (2) et (3). 
Pour ce faire les dérivées en espace (limitées dans notre cas à l'ordre 5) des opérateurs des équations (9), (10) et (11) sont discrétisées suivant un schéma centré à 7 points en suivant un développement de Taylor (ce qui implique que les dérivées premières et secondes sont discrétisées à l'ordre 6 alors que la dérivée cinquième l'est à l'ordre 2).

Le système d'équations (8) et (10) exprimé à la surface libre peut alors être écrit sous la forme matricielle suivante.

$$
\left.\left[\begin{array}{ll}
A_{1} & A_{2} \\
B_{1} & B_{2}
\end{array}\right] \begin{array}{l}
\hat{u}^{*} \\
\hat{w}^{*}
\end{array}\right]=\left[\begin{array}{l}
\widetilde{U} \\
0
\end{array}\right]
$$

Après résolution de ce système, on réinjecte les solutions dans l'équation (10) ce qui nous permet de déterminer $\widetilde{w}$ et avancer dans l'incrémentation en temps.

\section{III.2 Conditions limites}

Pour reproduire les parois verticales du domaine on impose une condition de Dirichlet pour la vitesse horizontale $u=0$ et des conditions de Neumann pour la surface libre et la vitesse verticale, $\frac{\partial \eta}{\partial x}=0$ et $\frac{\partial w}{\partial x}=0$. Ces conditions limites sont prises en compte par symétrie par rapport aux frontières. Ainsi les coefficients de discrétisation qui sortent du domaine sont réfléchis de manière paire pour $\eta$ et $w$ et de manière impaire pour $u$ ( $\eta$ et $w$ sont supposées symétriques par rapport aux murs et $u$ antisymétrique).

\section{III.3 Génération et absorption de la houle}

Pour la génération et l'absorption des vagues, des zones de relaxations ont été mises en place en entrée et en sortie du domaine. Ceci implique simplement une transition entre deux variables discrètes via la fonction : $f^{*}(i)=\operatorname{cr}(i) f_{1}(i)+(1-\operatorname{cr}(i)) f_{2}(i)$

où $\mathrm{i}$ représente l'indice en espace et cr une fonction variant progressivement de 0 à 1 (un exemple en $\sin ^{2}$ est présenté dans la figure 1 ).

Ainsi en entrée du domaine, sur une zone large d'une longueur d'onde (zone 1, figure 2), on impose progressivement une solution théorique ( $f_{1}$ représente la solution théorique, une houle de Stokes au $3{ }^{\text {eme }}$ ordre ou la forme optimisée d'une fonction de courant par exemple et $f_{2}=0$ ). Ensuite sur la même longueur une zone de transition (zone 2) permet de passer progressivement de la solution purement théorique imposée en entrée à la solution numérique (dans ce cas $f_{1}$ est la solution numérique donnée par le modèle et $f_{2}$ la solution théorique utilisée en entrée). Cette zone assure aussi l'absorption des champs de vagues réfléchis.

Et finalement dans le cas d'une absorption à la sortie du domaine (zone 3), on aura simplement $f_{1}=0$ et $f_{2}$ correspondant à la solution numérique.

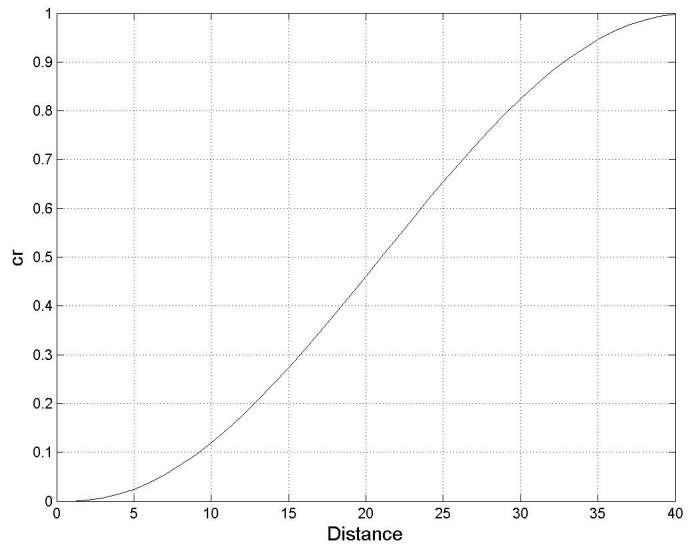

Figure 1 : Fonction cr utilisée

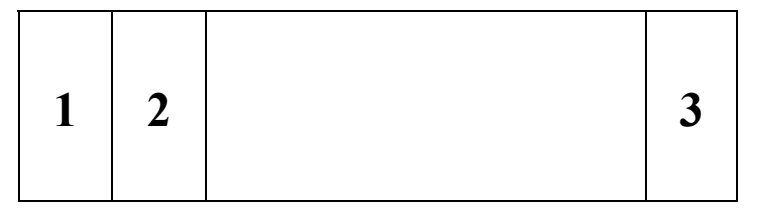

Figure 2: Zones de relaxation 


\section{IV - INTERACTION HOULE-COURANT}

\section{IV.1 Prise en compte du courant}

Les équations du modèle décrites précédemment permettent la propagation d'un courant sur un fond faiblement variable. Les seules modifications à effectuer sont sur les conditions limites qui doivent laisser passer le flux.

Ainsi à chaque extrémité du domaine on impose $u=U_{\text {courant }}$ au niveau de la paroi. $u$ devient alors antisymétrique par rapport à la valeur $U_{\text {courant }}$ (dans le cas sans courant, $U_{\text {courant }}=0$, on retrouve que $u$ est impaire au niveau du mur).

Dans les cas de la propagation d'un courant seul sur une bathymétrie variable, une première estimation de la solution peut être obtenue analytiquement grâce à l'équation de Bernoulli et une équation de conservation de flux, ce qui permet au modèle d'atteindre un état d'équilibre très rapidement au lieu d'attendre que le courant se propage sur tout le domaine. Par ailleurs on constate sans surprise que la solution d'équilibre est très peu différente de la solution initiale théorique.

Ainsi pour une bathymétrie $h$ et un courant en entrée du domaine $U_{0}$ donnés, les conditions initiales de surface libre et de courant sont données sur l'ensemble du domaine par la résolution du système où l'on a négligé la composante verticale du courant :

$$
\begin{aligned}
& \eta+\frac{U^{2}}{2 g}=\frac{U_{0}}{2 g} \\
& (\eta+h) U=h_{o} U_{o}
\end{aligned}
$$

Dans le cas d'un fond plat, la surface libre est laissée à z=0 et on impose seulement la valeur du courant sur tout le domaine.

\section{IV.2 Etude linéaire de la relation de dispersion}

Tout d'abord en suivant l'approche de Madsen et Schäffer [7], une étude théorique des propriétés linéaires de dispersion en présence de courant a été menée. A partir des équations (2), (3), (9) et (10) linéarisées on obtient la relation de dispersion suivante :

$$
\frac{\left(w-k U_{c}\right)^{2}}{g k}=\frac{\left(\kappa+\frac{1}{9} \kappa^{3}+\frac{1}{945} \kappa^{5}\right)}{\left(1+\frac{4}{9} \kappa^{2}+\frac{1}{63} \kappa^{4}\right)} \quad \text { (14) où } \kappa=k h .
$$

qui correspond à un développement de Padé $[4,4]$ de la relation théorique de Stokes au premier ordre :

$$
\frac{\left(w-k U_{c}\right)^{2}}{g k}=g k \tanh (\kappa)
$$

Le graphique suivant représente les solutions de ces équations en nombre d'onde adimensionnalisé $k h$ ( $h$ étant la profondeur d'eau), en fonction du nombre de Froude $F_{r}=U_{c} / \sqrt{g h}$ pour diverses profondeurs d'eau (exprimées en profondeur d'eau relative, $k_{0} h$ avec $k_{0}=w^{2} / g$ ). La fin de chaque courbe indique que les vagues sont bloquées par le courant. La relation de dispersion inhérente au modèle est donc très fidèle à la théorie de Stokes même pour des grandes profondeurs, cependant on constate que le blocage ne se produit pas pour des vagues très courtes et des courants faibles (non visible sur ce graphique). D'un point de vue physique cela signifie que d'après la théorie de Stokes les vagues courtes seront bloquées en présence d'un courant même faible alors que les solutions issues de la relation (14) continuent à se propager avec une longueur d'onde tendant vers zéro. 


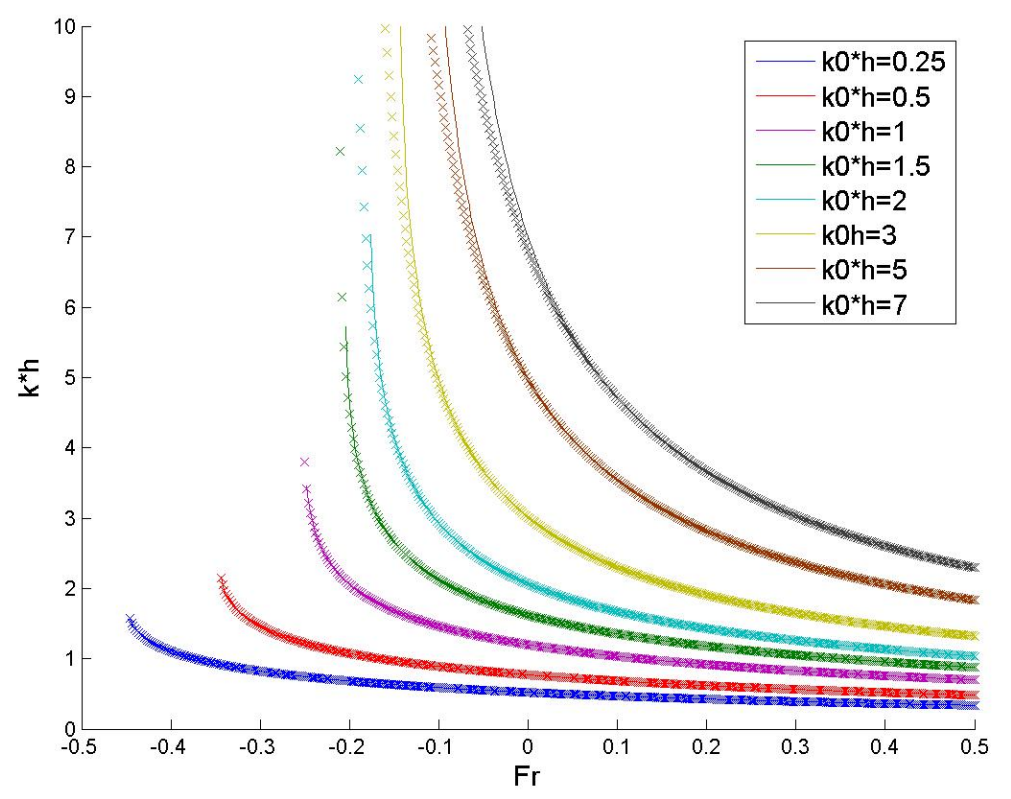

Figure 3: Solutions de la relation de dispersion en présence de courant pour différentes profondeur d'eau relatives $k_{o} h$, les lignes représentent la théorie de Stokes au premier ordre et les croix la relation théorique du modèle

\section{IV.3 Etude numérique}

Afin de vérifier l'analyse théorique précédente une étude numérique a été menée.

Une rampe douce est mise en place entre une zone de très grande profondeur (ou l'influence du courant sur la houle est alors négligeable) et une zone de profondeur souhaitée pour l'étude.

Ainsi dans l'exemple suivant (figure 4), l'étude porte sur la propagation d'une houle de période $10 \mathrm{~s}$ dans une profondeur d'eau de $10 \mathrm{~m}$ pour diverses valeurs de courant (dans ces conditions, $k_{0} h=0.4$ ).

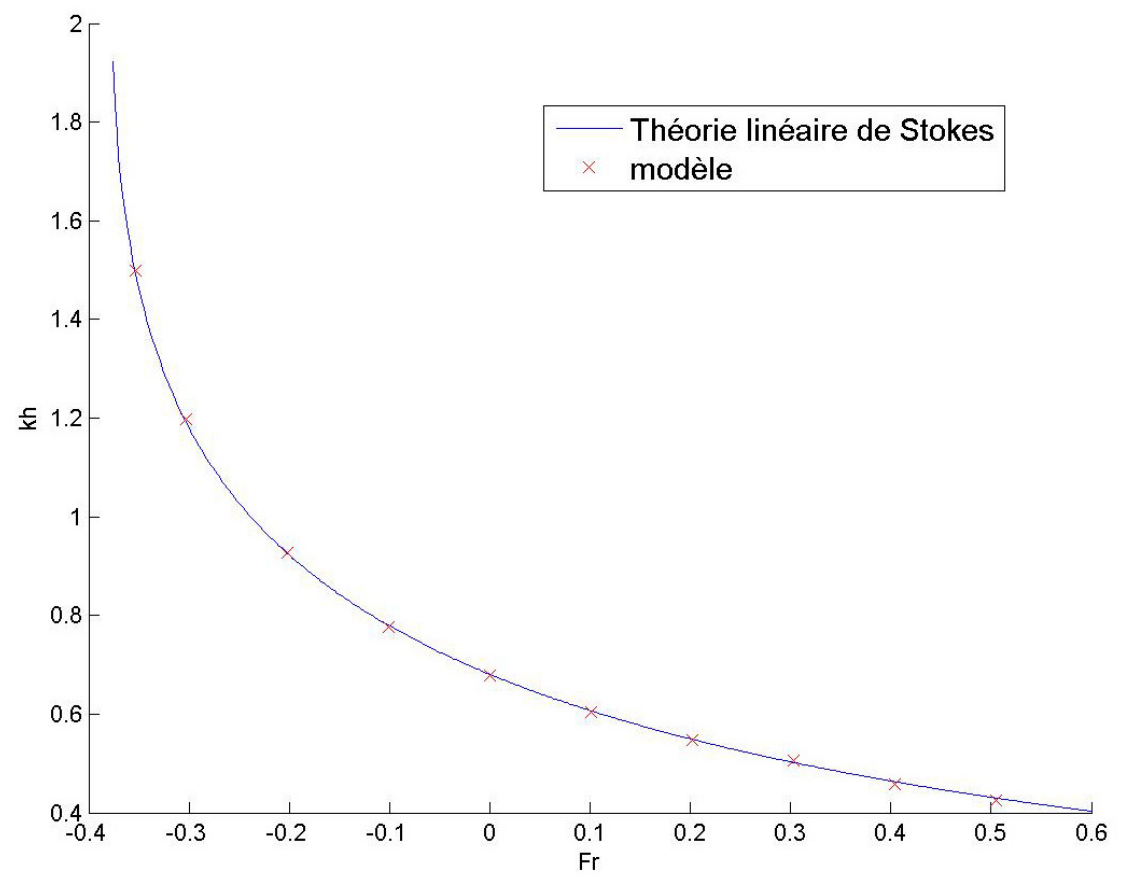

Figure 4 : Nombre d'ondes relatifs kh en fonction du nombre de Froude. 
Comme prévu on constate une très bonne corrélation entre résultats du modèle de Boussinesq et théorie de Stokes en faible profondeur d'eau. De manière générale, le modèle suit parfaitement la tendance présentée par l'étude théorique précédente (IV.3), avec un écart entre les valeurs du modèle et la théorie de Stokes croissant avec la profondeur d'eau.

\section{IV.4 Simulation de blocage des vagues}

Des simulations de blocages des vagues contre le courant inspirées de celles effectués par Madsen et Schäffer [7] ont été menées afin de vérifier le bon fonctionnement du code. Le graphique suivant représente la bathymétrie utilisée pour l'étude. La configuration est similaire à celle de Madsen et Schäffer [7] hormis le fait qu'une bathymétrie sinusoïdale a été préférée à une bathymétrie linéaire afin de limiter les perturbations au niveaux des bords (figure 5). Le canal fait $60 \mathrm{~m}$ de long pour une profondeur d'eau sur les bords de $0.8 \mathrm{~m}$ et de $0.2 \mathrm{~m}$ au sommet de la barre.

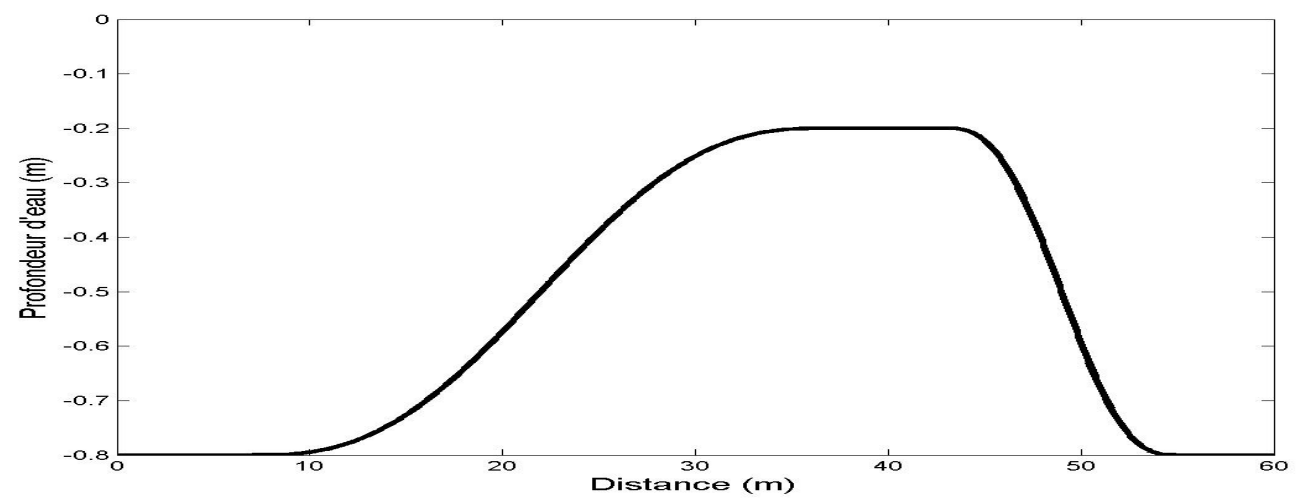

Figure 5 : Représentation de la bathymétrie utilisée pour étudier le blocage des vagues. Le courant se propage de la droite vers la gauche, les vagues dans l'autre sens.

On impose ensuite un courant uniforme de $-0.17 \mathrm{~m} / \mathrm{s}$ à la frontière droite du domaine. Ce courant converge au sommet de la bosse pour atteindre $-0.809 \mathrm{~m} / \mathrm{s}$.

Les variations d'élévation de surface libre et de vitesse de courant dues à la bathymétrie sont présentées ci-après. Au sommet de la bosse, la surface libre se situe à $-0.0319 \mathrm{~m}$.

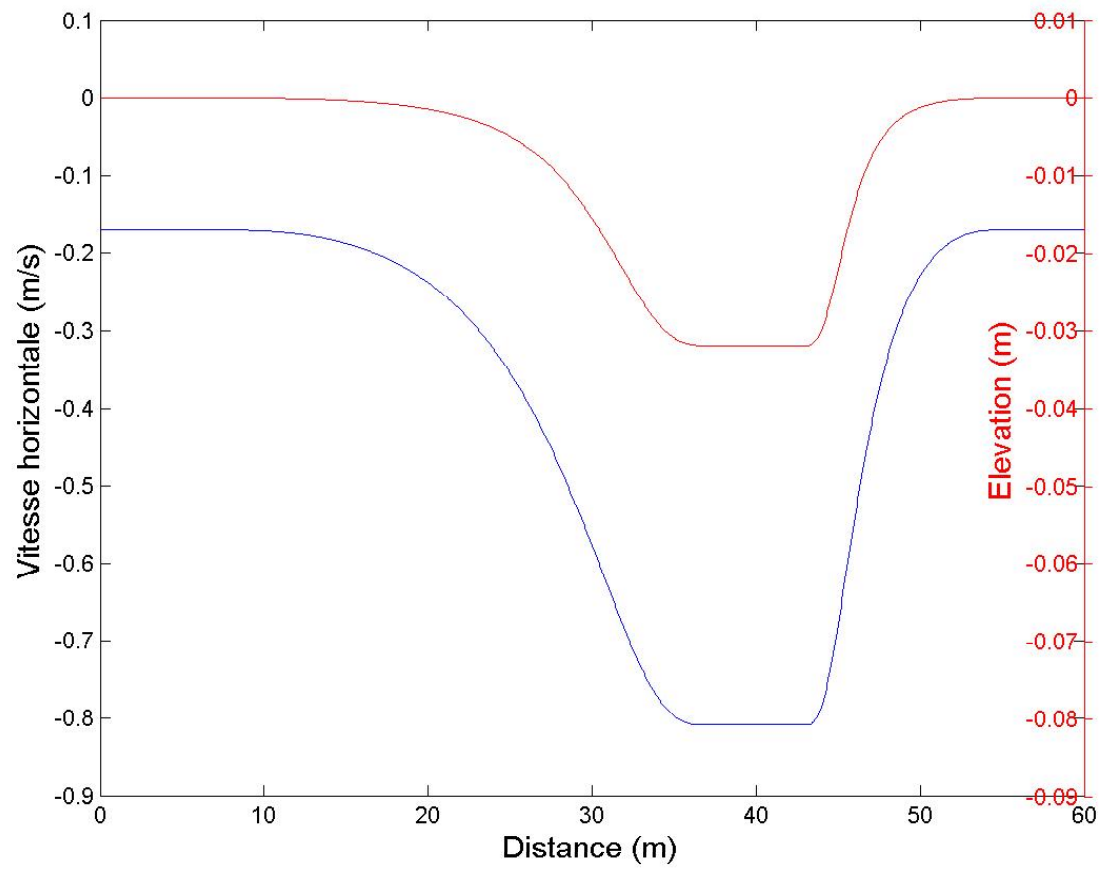

Figure 6 : Variations de vitesse horizontale (en noir) et de surface libre (en rouge) 
Enfin la houle est générée sur cet état d'équilibre à partir des zones de relaxations situées à l'entrée du domaine.

Dans l'exemple suivant la période de la houle est de $1.2 \mathrm{~s}$ avec une hauteur initiale de $0.02 \mathrm{~m}$. D'après la théorie linéaire de Stokes, on constate que cette houle sera bloquée pour $\mathrm{Fr}=$ 0.312 (valeur pour laquelle la relation de dispersion n'admet plus de solution), comme le montre la figure suivante qui représente en bleu la zone où la vitesse du courant bloque la propagation des vagues et en rouge l'évolution de la vitesse du courant en fonction de la bathymétrie dans notre cas test (toutes les valeurs étant adimensionnelles sur le schéma). Cette valeur de Fr est obtenue pour $\mathrm{x}=28,7 \mathrm{~m}$ dans notre configuration.

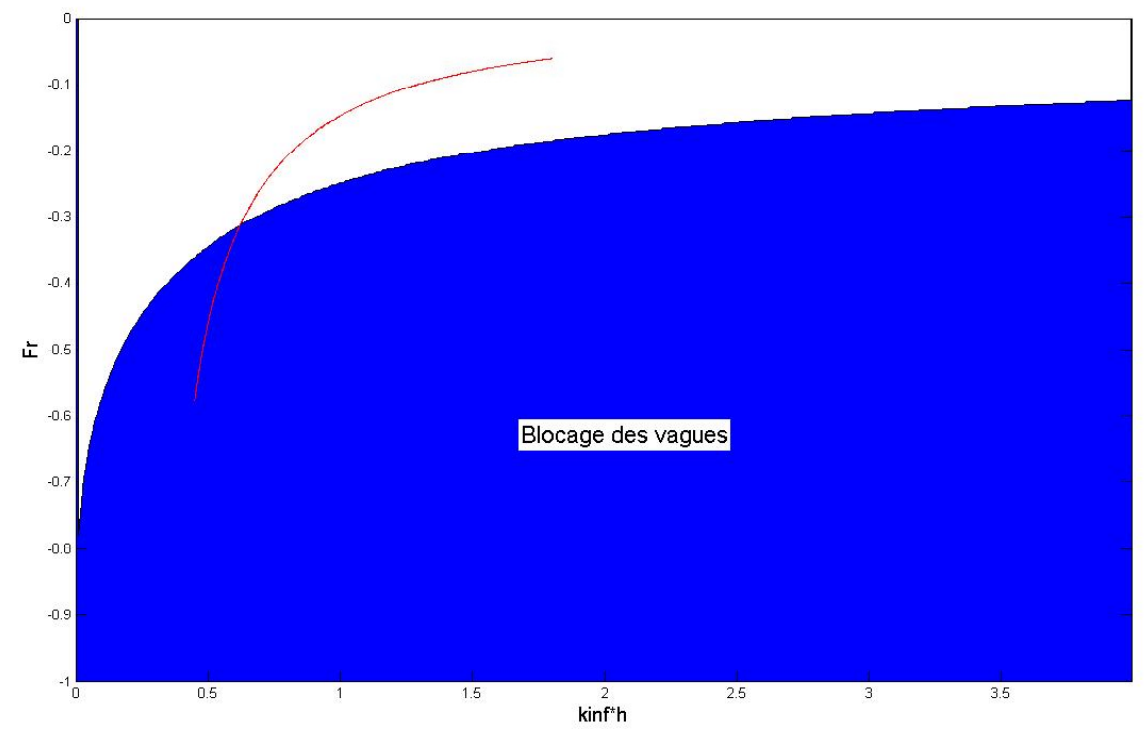

Figure 7: Valeurs du nombre de Froude correspondant au blocage des vagues pour différentes profondeurs relatives. En bleu, zone où la propagation est impossible et en rouge évolution du nombre de Froude dans la configuration étudiée (figures 5 et 6 )

La figure 8 montre l'élévation de la surface libre (sans la composante stationnaire due au courant).

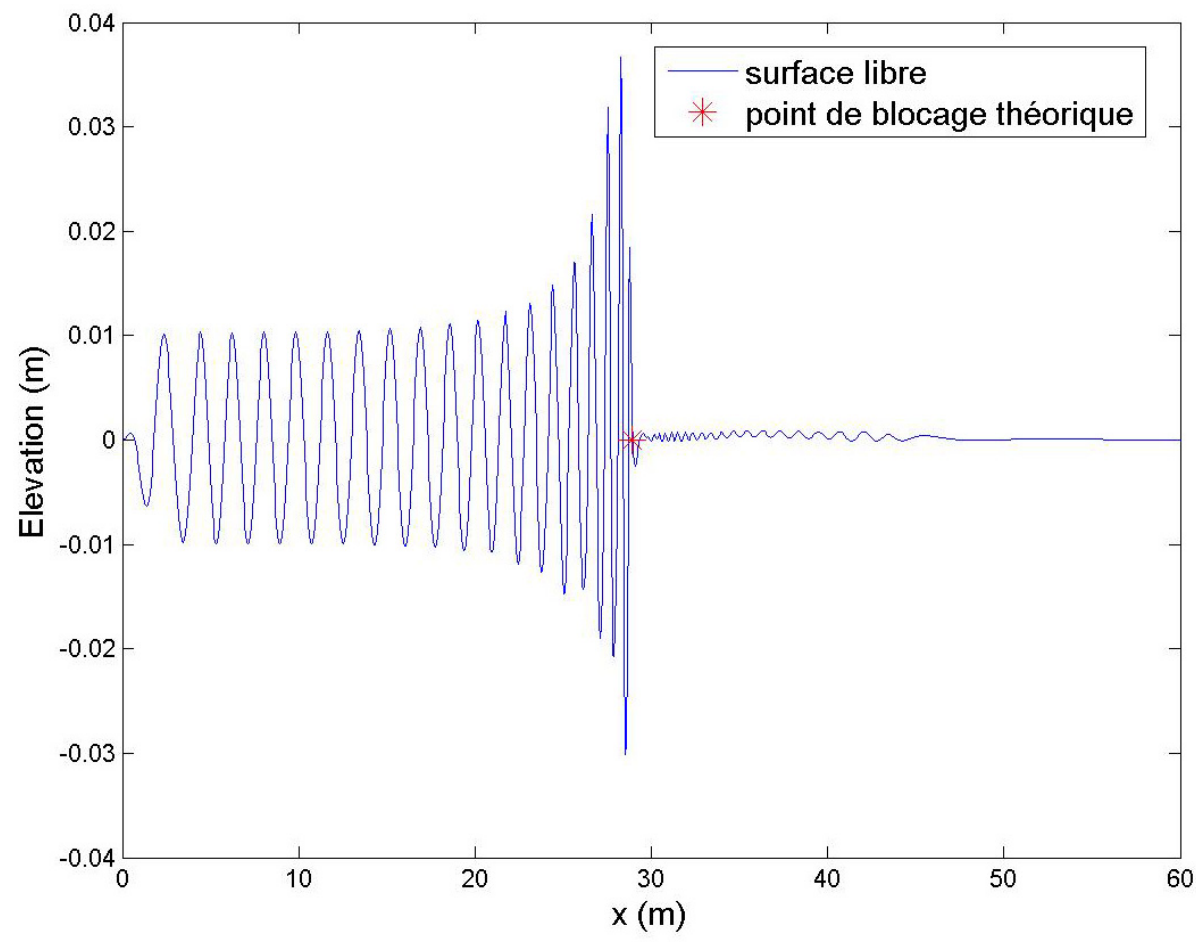

Figure 8 : Vagues bloquées par un courant opposé 
On observe que le champ de vague est bloqué à $\mathrm{x}=29.1 \mathrm{~m}$ ce qui représente une erreur d'environ $1 \%$ par rapport à la théorie.

Ainsi de manière générale le modèle est capable de modéliser avec une très bonne précision le blocage des vagues sauf pour des vagues courtes telles que $k_{0} h>1.5$.

\section{V - ETUDE DES PROFULS DE VITESSE}

\section{V.1 Etude théorique}

Les équations du modèle sont optimisées afin d'obtenir une précision maximale sur le profil des vitesses. La précision des profils des vitesses horizontale et verticale pour des cas linéaires et non-linéaires a fait l'objet d'une étude complète dans Madsen et Agnon [10] qui ne sera pas re-développée ici. Cependant il est important de noter qu'il ressort de cette étude que les profils de vitesses sont très fidèles aux théories existantes. Ainsi comparées aux solutions issues des fonctions de courant, l'erreur sur les distributions verticales de vitesse ne dépasse pas $2 \%$ pour $k h<16$ même pour des cas fortement non-linéaires.

\section{V.2 Reconstruction des profils de vitesse}

A partir des équations (9) et (10) on peux évaluer le champ de vitesse sur la colonne d'eau à chaque instant et à chaque point du domaine. En fond plat ces équations s'écrivent :

$$
\begin{aligned}
& u(x, y, z, t)=J_{01} \hat{u}^{*}+J_{02} \hat{w}^{*} \\
& w(x, y, z, t)=J_{01} \hat{w}^{*}-J_{02} \hat{u}^{*}
\end{aligned}
$$

Avec $J_{01}=1+\left(-\frac{\psi^{2}}{2}+\frac{\hat{z}^{2}}{18}\right) \nabla^{2}+\left(\frac{\psi^{4}}{24}-\frac{\hat{z}^{2} \psi^{2}}{36}+\frac{\hat{z}^{4}}{504}\right) \nabla^{4}$

$J_{02}=\psi+\left(-\frac{\psi^{3}}{6}+\frac{\hat{z}^{2} \psi}{18}\right) \nabla^{3}+\left(\frac{\psi^{5}}{120}-\frac{\hat{z}^{2} \psi^{3}}{108}+\frac{\hat{z}^{4} \psi}{504}\right) \nabla^{5}$ où $\psi \equiv z-\hat{z}$

Ci-dessous est représenté un exemple de représentation de profils verticaux de vitesse horizontale à différents instants (figure 9).

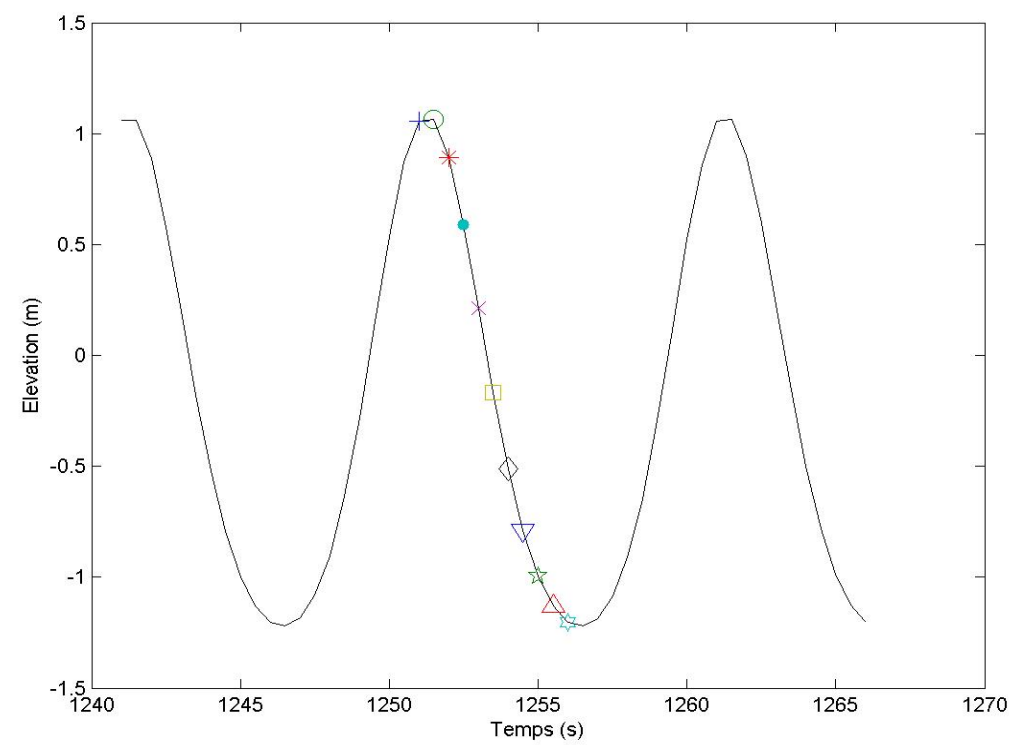




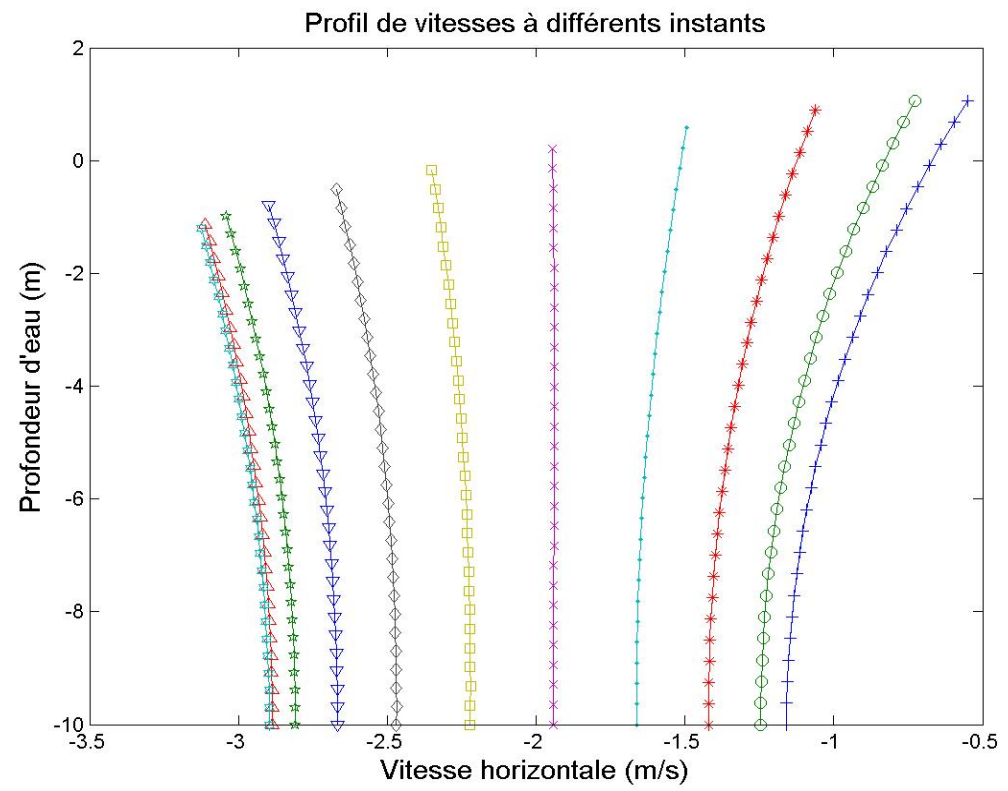

Figure 9 : Représentation des profils verticaux de vitesse horizontale, en haut localisation des points calculés et en bas les profils respectifs (les paramètres de la houle sont : hauteur crête à creux $=2 \mathrm{~m}$, période $=10 \mathrm{~s}$, profondeur d'eau $=10 \mathrm{~m}$ et vitesse du courant $=-1.8 \mathrm{~m} / \mathrm{s}$ ).

\section{V.3 Apparition du courant de retour :}

Pour contrebalancer le transport de masse créé par la houle seule, il y a un nécessairement apparition d'un courant de retour (cf Molin [11] par exemple). La vitesse moyennée de ce courant de retour dans la tranche d'eau vaut :

$$
U_{R}=-\frac{1}{2} \frac{a^{2} w}{h} \operatorname{coth}(k h)
$$

La présence de ce courant a été mise en évidence lors d'une étude des profils de courant issus du modèle. On observe un courant relativement constant sur la tranche d'eau opposé à la propagation des vagues dont la moyenne, sur l'ensemble des cas étudiés, vaut :

$$
\frac{U_{\text {modele }}}{U_{R}}=1 \pm 5 \%
$$

\section{$\mathrm{VI}$-Conclusion:}

Un modèle de type Boussinesq d'ordre élevé fondé sur une approche originale a été développé afin d'étudier les interactions houle-courant sur une bathymétrie quelconque. Il ressort que cette approche permet de représenter avec une grande précision les différents phénomènes liés à cette interaction (modification de la relation de dispersion de la houle, blocage des vagues...).

Le code doit être étendu au cas tridimensionnel avec un couplage à des champs incidents de houle aléatoire.

La description fine de la cinématique au travers des profils de vitesse permettra à terme de définir les conditions d'entrée de dispositifs convertisseurs d'énergie des courants en présence de houle ou de sous-domaines dans lesquels des approches en fluide visqueux pourraient être conduites. 


\section{REEERENCES}

[1] Phase-decoupled refraction-diffraction for spectral wave models Coastal Engineering, Volume 49, Issue 4, October 2003, Pages 291-305

L. H. Holthuijsen, A. Herman and N. Booij

[2] A three-dimensional multi-level turbulence model for tidal motion Ocean Engineering, Volume 26, Issue 11, November 1999, pp. 1023-1038

Xiaobo Chao, N. J. Shankar and Cheong Hin Fatt

[3] Effects of surface waves on a turbulent current over a smooth or rough seabed ZHENHUA HUANG, CHIANG C. MEI

Journal of Fluid Mechanics, Volume 497,. December 2003. pp253-287

[4] Estimates of wave decay rates in the presence of turbulent currents Applied Ocean Research, Volume 23, Issue 3, June 2001, pp. 125-137

L. Thais, G. Chapalain, G. Klopman, R. R. Simons and G. P. Thomas

[5] Stability of periodic waves of finite amplitude on the surface of a deep fluid J. Appl. Mech. Tech. Phys., 1968, Vol. 9, pp. 190-194

V.E. Zakharov

[6] A new Boussinesq method for fully nonlinear waves from shallow to deep water.

J. Fluid Mech. (2002), vol. 462, pp.1-30

P.A. Madsen, H.B. Bingham and Hua Liu

[7] A review of Boussinesq-type equations for surface gravity waves

Advances in Coastal and Ocean Engineering (1999) Vol.5

P.A. Madsen and H.A. Schäffer

[8] Numerical solutions of Boussinesq equations for fully nonlinear and extremely dispersive water waves

PhD Thesis, (2004)

D.R. Fuhrman

[9] A Boussinesq-type method for fully nonlinear waves interacting with a rapidly varying bathymetry

Coastal Engineering, Volume 53, Issues 5-6, April 2006, Pages 487-504

P.A. Madsen, D.R. Furhman, B. Wang

[10] Accuracy and convergence of velocity formulations for water waves in the framework of Boussinesq theory

J. Fluid Mech. (2003), vol. 477, pp.285-319

P.A. Madsen and R. Y. Agnon

[11] Hydrodynamique des structure offshore

Bernard Molin

[12] Etude hydrodynamique d'un type d'hydraulienne à axe vertical pour les courants marins Thèse de Doctorat de l'INP Grenoble, LEGI, sous la direction de M. J.L. Achard Cristina Ploesteanu 